\title{
EFEITO AGUDO DA CORRIDA COM OS PÉS DESCALÇO SOBRE AS COMPONENTES ANTEROPOSTERIOR E MEDIOLATERAL DA FORÇA DE REAÇÃO DO SOLO
}

\author{
SHORT-TERM EFFECTS OF BAREFOOT RUNNING ON \\ ANTEROPOSTERIOR AND MEDIOLATERAL COMPONENTS OF \\ GROUND REACTION FORCE
}

\section{EFECTO AGUDO DEL CORRER DESCALZO SOBRE LAS COMPONENTES ANTEROPOSTERIOR Y MEDIOLATERAL DE LA FUERZA DE REACCIÓN DEL SUELO}

\author{
Ana Paula da Silva Azevedo \\ https://orcid.org/0000-0002-0430-8040 (D) \\ http://lattes.cnpq.br/0987782639429253 9 \\ Universidade de São Paulo (São Paulo, SP - Brasil) \\ anaazevedo@usp.br \\ Carlos Alberto Cardoso Filho \\ https://orcid.org/0000-0003-3204-9397 (DD \\ http://lattes.cnpq.br/0248219632673942 9 \\ Universidade de São Paulo (São Paulo, SP - Brasil) \\ carlos.filho@alumni.usp.br \\ Wilson Pereira Lima \\ https://orcid.org/0000-0002-1832-5108 (D) \\ http://lattes.cnpq.br/9144131870576428 9 \\ Universidade de São Paulo (São Paulo, SP - Brasil) \\ wplima22@gmail.com \\ Maura de Arruda Botelho Colturato \\ https://orcid.org/0000-0003-3649-5060 (iD \\ http://lattes.cnpq.br/3635399924473627 9 \\ Universidade de São Paulo (São Paulo, SP - Brasil) \\ mauracolturato@gmail.com \\ Júlio Cerca Serrão \\ https://orcid.org/0000-0002-3646-3387 (D) \\ http://lattes.cnpq.br/9124685212860479 \\ Universidade de São Paulo (São Paulo, SP - Brasil) \\ jcserrao@usp.br
}

\section{Resumo}

Este estudo investigou o efeito agudo da corrida descalço sobre parâmetros selecionados das componentes anteroposterior e mediolateral da Força de Reação do Solo (FRS) em corredores habituados ao uso do calçado esportivo. Setenta e um corredores recreacionais, inexperientes em corrida descalço, correram em velocidade autoselecionada por uma distância de 9 metros em piso fixo equipado com plataformas de força, sob duas condições: calçados e descalços. Os picos de frenagem e aceleração da componente anteroposterior, bem como os picos 
mínimo e máximo da componente mediolateral foram obtidos. O pico de frenagem foi menor, enquanto o pico de aceleração foi maior na corrida descalço. Em contrapartida, a magnitude de ambos os picos mínimo e máximo foram maiores na condição descalço. Em síntese, a corrida descalço altera agudamente parâmetros selecionados das componentes anteroposterior e mediolateral da FRS relacionados à sobrecarga e ao desempenho de corredores habituados ao uso do calçado esportivo.

Palavras-chave: Locomoção; Biomecânica; Sobrecarga; Desempenho.

\section{Abstract}

This study investigated the short-term effects of barefoot running on selected parameters of the anteroposterior and mediolateral components of the Ground Reaction Force (GRF) in runners used to wearing athletic shoes. Seventy-one recreational runners, inexperienced in barefoot running, ran at a self-selected speed for a distance of 9 meters on a fixed floor equipped with force platforms, under two conditions: shod and barefoot. The braking and acceleration peaks of the anteroposterior component, as well as the minimum and maximum peaks of the mediolateral component were obtained. The braking peak was lower, while the acceleration peak was higher in barefoot running. In contrast, the magnitude of both the minimum and maximum peaks were greater in the barefoot condition. In summary, barefoot running acutely alters selected parameters of the anteroposterior and mediolateral components of the FRS related to the overload and performance of runners used to wearing sports shoes.

Keywords: Locomotion; Biomechanics; Overload; Performance.

\section{Resumen}

Este estudio investigó el efecto agudo de correr descalzo en parámetros seleccionados de los componentes anteroposterior y mediolateral de la fuerza de reacción del suelo (FRS) en corredores acostumbrados a usar calzado deportivo. Setenta y un corredores recreativos, sin experiencia en correr descalzo, corrieron a una velocidad autoseleccionada por una distancia de 30 pies en un piso fijo equipado con plataformas de fuerza, bajo dos condiciones: zapatos y descalzo. Se obtuvieron los picos de frenado y aceleración de la componente anteroposterior, así como los picos mínimos y máximos de la componente mediolateral. El pico de frenado fue menor, mientras que el pico de aceleración fue mayor al correr descalzo. Por el contrario, la magnitud de los picos tanto mínimo como máximo fue mayor en la condición de descalzo. En resumen, correr descalzo altera de forma aguda parámetros seleccionados de los componentes anteroposterior y mediolateral del FRS relacionados con la sobrecarga y el rendimiento de los corredores acostumbrados a llevar calzado deportivo.

Palabras clave: Locomoción; Biomecánica; Sobrecarga; Actuación.

\section{INTRODUÇÃO}

A corrida com os pés descalços tem sido apontada como uma estratégia de locomoção potencialmente capaz de alterar a técnica de movimento, reduzir as forças externas e, consequentemente, diminuir os riscos de lesões em corredores (LIEBERMAN et al., 2010a; ALTMAN; DAVIS, 2012; 2016; DAOUD et al., 2012; WILLY; DAVIS, 2014; DA SILVA AZEVEDO et al., 2016a; HOLLANDER et al., 2019). Considerando-se especificamente a Força de Reação do Solo (FRS), principal força externa atuante durante a corrida, há evidências de que importantes variáveis da sua componente vertical relacionadas ao controle de choque mecânico sofrem a influência da condição descalço (DIVERT et al., 2005; LIEBERMAN et al., 2010b; WILLY; DAVIS, 2014; DA SILVA AZEVEDO et al., 2016a, 2016b; HOLLANDER et al., 2019). Enquanto a literatura reporta aumentos no pico passivo (Fy1) e taxa de desenvolvimento da força ao longo desse pico (TDF1) como efeitos agudos da corrida descalço (WIT; CLERCQ; AERTS, 2000; LOHMAN;

BALAN SACKIRIYAS; SWEN, 2011; FLEMING et al., 2015), sugerindo aumento do impacto 
recebido, outros estudos apontam a redução dessas mesmas variáveis em resposta ao uso crônico da corrida descalça, sugerindo a possibilidade do aparelho locomotor se adaptar e aperfeiçoar seu controle de impacto sob essa condição (LIEBERMAN et al., 2010b; WARNE et al., 2013; SAMAAN; RAINBOW; DAVIS, 2014; WARNE; WARRINGTON, 2014; KHOWAILED et al., 2015; DA SILVA AZEVEDO et al., 2016a, 2016b; HOLLANDER et al., 2019).

O fato do transiente de impacto da componente vertical da FRS e sua taxa de desenvolvimento estarem associados a diversas lesões relacionadas à corrida, como fraturas por estresse, dor patelofemoral e fasciite plantar (MILNER et al., 2006; SARAGIOTTO et al., 2014; DAVIS; BOWSER; MULLINEAUX, 2016; SUN et al., 2020), tem suscitado concentração dos estudos acerca desta temática na componente vertical da FRS, havendo poucos dados sobre o comportamento das componentes anteroposterior e mediolateral dessa força diante da adoção da corrida com os pés descalços. Embora apresentem magnitudes significativamente menores que as observadas para a componente vertical, as componentes anteroposterior e mediolateral podem fornecer valiosas informações acerca do movimento, sobretudo relacionadas ao controle de sobrecarga e desempenho na corrida.

A componente anteroposterior da FRS (Fx) apresenta, em média, magnitude de 0,5 PC (peso corporal), refletindo a aceleração horizontal do indivíduo (na direção do movimento) e as forças geradas em resposta ao comportamento dessa aceleração durante a fase de apoio (HAMILL; KNUTZEN; DERRICK, 2015). Esta componente é caracterizada por um pico de frenagem no início do apoio, relacionado às forças de fricção exercidas contra o corredor e absorvidas durante a desaceleração; e por um pico de aceleração no final do apoio, representando as forças propulsivas geradas pelo corredor na direção do movimento (HAMILL; KNUTZEN; DERRICK, 2015). Considerando-se que a minimização do pico de frenagem e maximização do pico de aceleração poderia indicar menores sobrecargas sobre as estruturas no eixo anteroposterior e menor desaceleração do corredor, a investigação dos efeitos da corrida descalço sobre esses componentes contribuiria para maior entendimento do controle de sobrecarga e desempenho na corrida (HUNTER; MARSHALL; MCNAIR, 2005; HAMILL; KNUTZEN; DERRICK, 2015).

A componente mediolateral da FRS (Fz) é um pouco mais variável e menos consistente entre os indivíduos, apresentando magnitude média de 0,1 PC, e representa as forças geradas de um lado a outro do pé em resposta a aceleração neste eixo ao longo da fase de apoio (HAMILL; KNUTZEN; DERRICK, 2015). Considerando que esta componente está 
relacionada aos movimentos de pronação e supinação (HAMILL; KNUTZEN; DERRICK, 2015), alterações na componente mediolateral podem induzir a movimentos articulares excessivos no tornozelo. Sabendo-se que movimentos excessivos de pronação e supinação são associados a dores na perna e joelho de corredores (WILLEMS et al., 2006; RODRIGUES; TENBROEK; HAMILL, 2013) $(23,24)$, acessar o efeito da corrida descalço sobre essa componente torna-se relevante.

Diante da potencial contribuição das componentes anteroposterior e mediolateral no entendimento da sobrecarga e desempenho na corrida, e considerando a escassez de dados acerca do comportamento destes parâmetros durante a corrida descalço, o propósito deste estudo foi verificar o efeito agudo da corrida com os pés descalços sobre variáveis selecionadas das componentes anteroposterior e mediolateral da FRS de corredores habituados ao uso do calçado esportivo. Mais especificamente, o estudo investigou o efeito da corrida descalço sobre os picos de frenagem e aceleração da componente anteroposterior e sobre os picos mínimo e máximo da componente mediolateral da FRS de corredores inexperientes nesta condição de corrida.

\section{MATERIAIS E MÉTODOS}

Setenta e um corredores recreacionais habituados ao uso do calçado esportivo (26 homens e 45 mulheres; $28.41 \pm 4.87$ anos; $62.48 \pm 24.33 \mathrm{~kg} ; 1.66 \pm 0.09 \mathrm{~m}$ ) foram recrutados para participar do estudo. Um questionário prévio foi aplicado a fim de obter informações acerca da experiência em corrida, volume semanal de treino e lesões prévias nos membros inferiores. Para inclusão, os participantes deveriam ter idade entre 18 e 40 anos, possuir alguma experiência na prática da corrida, não estarem acometidos por nenhuma lesão ortopédica e/ou problema de saúde, nem apresentar histórico de lesão nos últimos 12 meses. Adicionalmente, não possuir experiência anterior em corrida com proteção plantar reduzida (descalço ou com calçados minimalistas) era também um critério fundamental para inclusão no estudo. Participantes que apresentaram problema de saúde, histórico de lesão nos últimos 12 meses, e/ou experiência anterior em corrida com proteção plantar reduzida foram excluídos do estudo. Todos os participantes foram informados sobre os objetivos e procedimentos do estudo, assinando o termo de consentimento informado. O desenho experimental do presente estudo foi aprovado pelo comitê de ética local. 
Cada participante foi avaliado em uma única sessão de teste. A primeira etapa correspondeu à aferição e mensuração de dados antropométricos (como massa e estatura). Em seguida, um período de 5 minutos de aquecimento e adaptação ao ambiente foi fornecido aos participantes. Ao término da familiarização, os participantes executaram corridas intermitentes, em velocidade auto-selecionada, por uma distância de 9 metros sobre um piso fixo equipado com duas plataformas de força. Os participantes corriam até que o número de tentativas válidas necessário fosse obtido (totalizando, em média, 20 minutos de teste). Os participantes eram instruídos a adotar uma velocidade confortável, que pudesse ser mantida em uma hipotética situação de 30 minutos de corrida. Esse procedimento foi realizado para a corrida com o uso do calçado esportivo habitual do participante e para a corrida com os pés descalços, na mesma sessão de teste, havendo 2 minutos de intervalo entre cada uma das condições. A ordem das condições experimentais foi determinada de maneira randomizada. Parâmetros relacionados às componentes anteroposterior (horizontal) e mediolateral da Força de Reação do Solo (FRS) foram obtidos (a $3000 \mathrm{~Hz}$ ) por intermédio de duas plataformas de força do tipo "strain gauge" (AMTI BP600900 - 2000; Watertown, EUA) posicionadas na segunda metade da distância de $9 \mathrm{~m}$.

Rotinas matemáticas em ambiente Matlab foram utilizadas para o processamento e tratamento matemático dos dados. Inicialmente, os dados de FRS foram processados a partir de um filtro Butterworth de $4^{\mathrm{a}}$ ordem, com frequência de corte de $90 \mathrm{~Hz}$. O início e fim de cada apoio foi determinado utilizando-se um limiar de 20 N. Os dados de FRS foram normalizados pelo peso corporal dos indivíduos, enquanto o tempo foi normalizado pelo tempo total de apoio. A partir desta análise, foram obtidos os seguintes parâmetros da FRS: pico máximo (FzMáx) e pico mínimo (FzMín) da componente mediolateral, relacionados à atenuação de choque mecânico neste eixo; pico da componente anteroposterior durante a fase de frenagem (Fx1_Fren), relacionado à desaceleração do corpo e atenuação de choque nesse eixo durante a fase inicial de contato; pico da componente anteroposterior durante a fase propulsiva (Fx2_Prop), relacionado à força produzida nesse eixo para aceleração do corpo à frente durante a fase propulsiva (fase final) do contato.

Os dados foram submetidos ao teste de normalidade D'Agostino-Pearson Omnibus, constatando-se a distribuição normal dos dados. A comparação entre as condições experimentais (Calçado x Descalço) foi feita a partir da aplicação do Teste T Pareado. Os dados 
estão expressos em média \pm DP. O nível de significância adotado foi $p<0.05$. O software GraphPad Prism 6.0 foi adotado para a análise estatística dos dados.

\section{RESULTADOS}

Diferenças significativas foram observadas entre as condições Calçado e Descalço para todas as variáveis analisadas. Com relação à componente anteroposterior, o pico de frenagem (Fx1_Fren) apresentou-se 2.5\% menos negativo $(p=0.033)$ para a condição Descalço, enquanto o pico de aceleração (Fx2_Prop) foi $11.2 \%$ maior $(p<0.001)$ para a corrida nesta mesma condição (Gráfico 1). A componente mediolateral apresentou pico máximo (FzMáx) 4.8\% maior ( $p=0.006$ ) e pico mínimo (FzMín) 13.2\% mais negativo ( $p=0.0001)$ para a corrida Descalço (Gráfico 2).

Gráfico 1 - Média e desvio padrão dos picos de frenagem (gráfico superior) e aceleração (gráfico inferior) da componente anteroposterior da Força de Reação do Solo (FRS), onde (*) indica diferença significativa entre as condições experimentais de corrida (Calçado e Descalço).
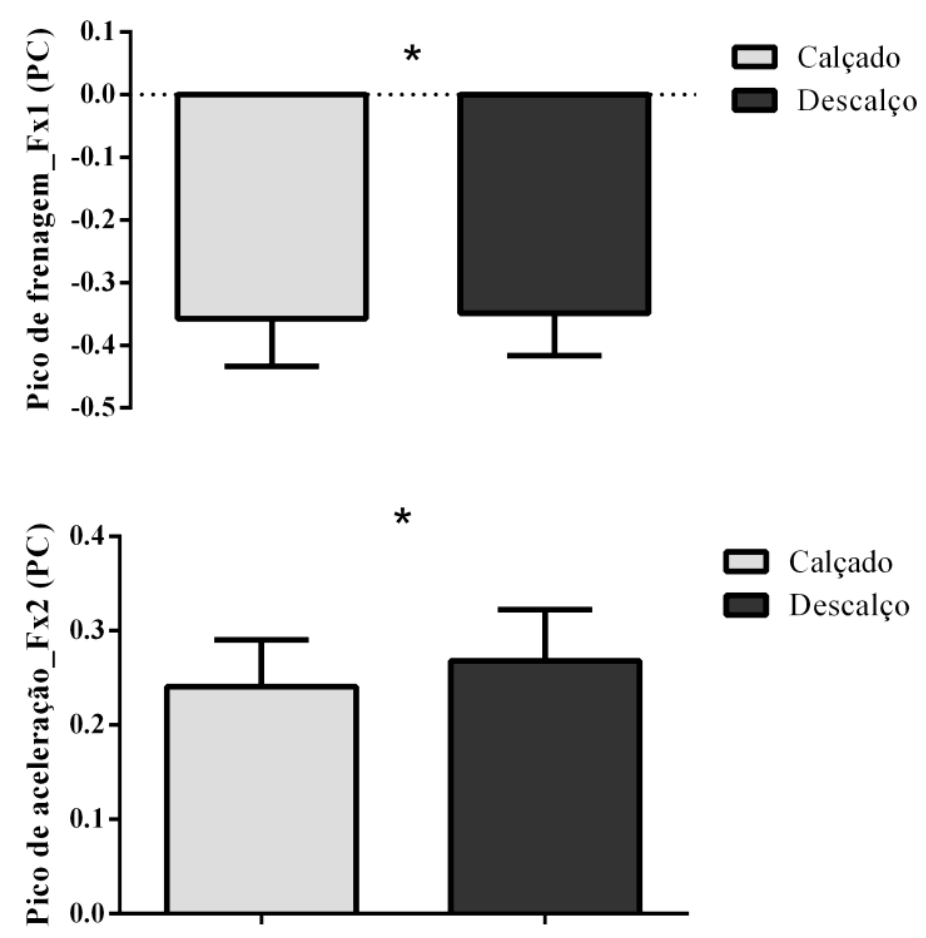

Fonte: Construção dos autores. 
Gráfico 2 - Média e desvio padrão dos picos máximo (gráfico superior) e mínimo (gráfico inferior) da componente mediolateral da Força de Reação do Solo (FRS), onde $\left(^{\star}\right)$ indica diferença significativa entre as condições experimentais de corrida (Calçado e Descalço).

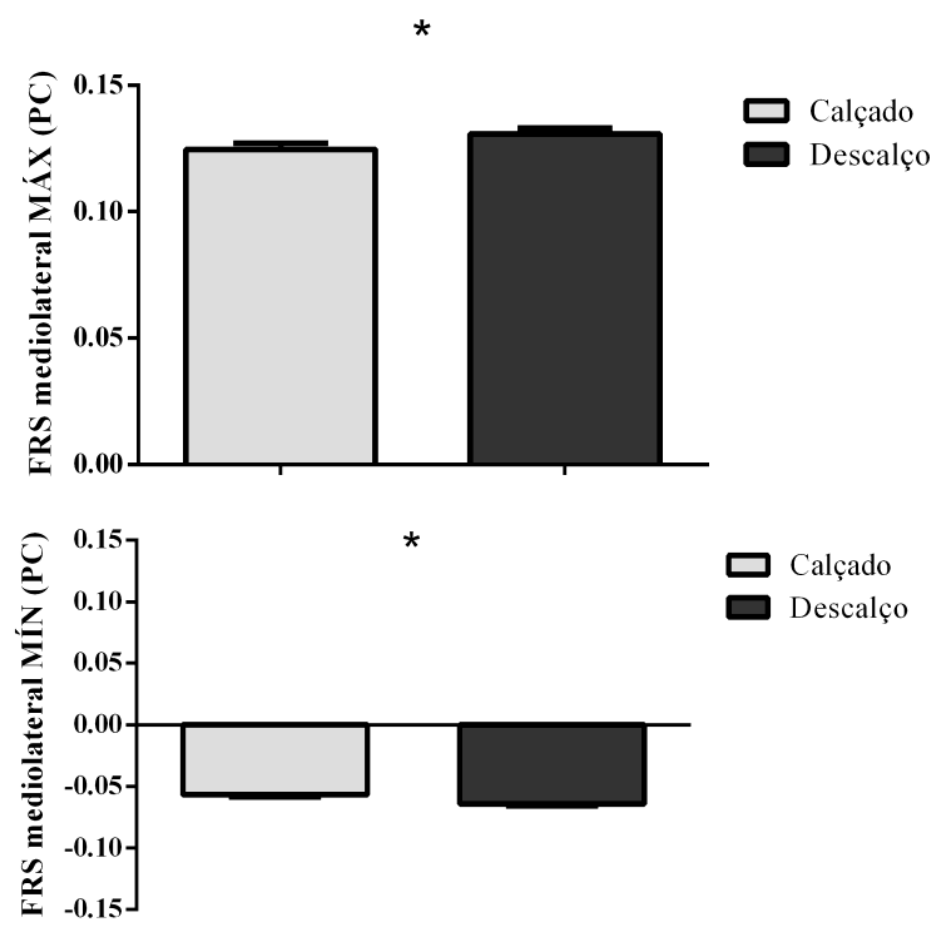

Fonte: Construção dos autores.

\section{DISCUSSÃo}

O presente estudo investigou o efeito da corrida descalço sobre parâmetros selecionados das componentes anteroposterior (horizontal) e mediolateral da Força de Reação do Solo (FRS) em corredores habituados ao uso do calçado esportivo. Dentro do melhor do nosso conhecimento, este é um dos poucos estudos que verificou a influência aguda da corrida descalço sobre as componentes anteroposterior e mediolateral da FRS.

Enquanto em estudo anterior não foram observadas diferenças significativas entre as condições calçado e descalço para os picos anteroposterior e mediolateral durante a corrida (THOMPSON; SEEGMILLER; MCGOWAN, 2016), como principal resultado deste trabalho verificou-se que a corrida com os pés descalços influencia agudamente parâmetros selecionados das componentes anteroposterior e mediolateral da FRS relacionados à sobrecarga e ao desempenho na corrida. O pico de frenagem menos negativo para a condição descalço sugere ligeira diminuição na sobrecarga anteroposterior a ser atenuada no início do contato, podendo significar menores riscos ao corredor (RODRIGUES; TENBROEK; HAMILL, 
2013; HAMILL; KNUTZEN; DERRICK, 2015). Adicionalmente, este resultado aponta uma menor desaceleração do indivíduo nesta fase da corrida, o que poderia contribuir para uma melhor performance (HUNTER; MARSHALL; MCNAIR, 2005). Corroborando a este resultado, o maior pico de aceleração para a corrida descalço sugere a geração de maiores forças propulsivas pelo corredor na direção do movimento sob esta circunstância, o que poderia induzir a uma melhor performance, especialmente em situações de "sprint" (HUNTER; MARSHALL; MCNAIR, 2005; HAMILL; KNUTZEN; DERRICK, 2015). Ao observar-se estes resultados em conjunto, percebe-se que o fato dos picos de frenagem e aceleração não serem idênticos em magnitude indica que velocidade média dos corredores não foi constante ao longo do teste, e há uma diferença entre o quanto se desacelera no início do contato e o quanto se propulsiona no final do contato (HAMILL; KNUTZEN; DERRICK, 2015). Sobretudo para a condição descalço, essa diferença parece gerar um saldo positivo para a aceleração, beneficiando o desempenho na tarefa (HUNTER; MARSHALL; MCNAIR, 2005).

Por outro lado, maiores picos mínimo e máximo da componente mediolateral foram observados para a corrida descalço. Tais resultados sugerem maiores sobrecargas no eixo mediolateral na corrida aguda sob esta condição, o que pode relacionar-se com maiores amplitudes de movimentos no tornozelo, como pronação e supinação (HAMILL; KNUTZEN; DERRICK, 2015). Sabendo-se que movimentos excessivos de pronação e supinação são associados a dores na perna e joelho de corredores, tais resultados podem indicar um possível efeito negativo da corrida descalço sobre essa componente (WILLEMS et al., 2005; RODRIGUES; TENBROEK; HAMILL, 2013) $(23,25)$.

Apesar da relevância do estudo, algumas limitações devem ser consideradas ao interpretar os presentes resultados. Primeiramente, nossos resultados são protocolodependentes e devem ser extrapolados com cautela para outras situações. Outras populações e/ou diferentes circunstâncias podem gerar resultados distintos. Segundo, os mecanismos por trás das mudanças observadas não foram acessados. Diante disso, mais estudos acerca dos efeitos da condição descalço sobre a corrida se fazem necessários para melhor compreensão deste fenômeno. Adicionalmente, o estudo acessou o efeito agudo da corrida descalço sobre a FRS. Sendo assim, se fazem também necessários estudos que investiguem os efeitos do uso crônico da condição descalço na FRS durante corrida, sobretudo nas componentes anteroposterior e mediolateral. 


\section{CONCLUSÃO}

A corrida com os pés descalços altera parâmetros selecionados das componentes anteroposterior e mediolateral da FRS em corredores habituados ao uso do calçado esportivo. De forma aguda, a corrida descalço parece diminuir ligeiramente as forças de frenagem e aumentar o potencial propulsivo no eixo anteroposterior, influenciado positivamente o controle de sobrecarga e desempenho. Por outro lado, as magnitudes dos picos de força mediolaterais apresentam-se aumentadas na corrida com os pés descalços, o que pode acarretar movimentos excessivos na articulação do tornozelo e aumentar o risco de lesões.

\section{REFERÊNCIAS BIBLIOGRÁFICAS}

ALTMAN, Allison R.; DAVIS, Irene S. Barefoot running: Biomechanics and implications for running injuries. Current sports medicine reports, v. 11, n. 5, p. 244-250, 2012.

Prospective comparison of running injuries between shod and barefoot runners. British journal of sports medicine, v. 50, n. 8, p. 1-6, 2016.

AZEVEDO, Ana Paula da Silva e colaboradores. 16 weeks of progressive barefoot running training changes impact force and muscle activation in habitual shod runners. PLoS ONE, $\mathrm{V}$. 11, n. 12, 2016.

AZEVEDO, Ana Paula da Silva e colaboradores. Does "transition shoe" promote an intermediate biomechanical condition compared to running in conventional shoe and in reduced protection condition? Gait and posture, v. 46, p. 142-146, 2016.

DAOUD, Adam I. e colaboradores. Foot strike and injury rates in endurance runners: a retrospective study. Medicine and science in sports and exercise, v. 44, p. 1325-1334, 2012.

DAVIS, Irene S.; BOWSER, Bradley J.; MULLINEAUX, David R. Greater vertical impact loading in female runners with medically diagnosed injuries: a prospective investigation. British journal of sports medicine, v. 50, n. 14, p. 887-892, 2016.

DIVERT, Carolyn e colaboradores. Mechanical comparison of barefoot and shod running. International journal of sports medicine, v. 26, n. 7, p. 593-598, 2005.

FLEMING, Neil e colaboradores. Acute response to barefoot running in habitually shod males. Human movement science, v. 42, p. 27-37, 2015.

HAMILL, Joseph; KNUTZEN, Kathleen M.; DERRICK, Timothy R. Biomechanical basis of human movement. 4th ed. Philadelphia: [s. n.], 2015.

HOLLANDER, Karsten e colaboradores. Adaptation of running biomechanics to repeated 
barefoot running: a randomized controlled study. American journal of sports medicine, v. 47, n. 8, p. 1975-1983, 2019.

HUNTER, Joseph P.; MARSHALL, Robert N.; MCNAIR, Peter J. Relationships between ground reaction force impulse and kinematics of sprint-running acceleration. Journal of applied biomechanics, v. 21, n. 1, p. 31-34,2005.

KHOWAILED, Iman A. e colaboradores. Six weeks habituation of simulated barefoot running induces neuromuscular adaptations and changes in foot strike patterns in female runners. Medical science monitor, v. 21, p. 2021-2030, 2015.

LIEBERMAN, Daniel E. e colaboradoresFoot strike patterns and collision forces in habitually barefoot versus shod runners. Nature, v. 463, n. 7280, p. 531-535, 2010.

LOHMAN, Everett B.; BALAN SACKIRIYAS, Kanikkai Steni; SWEN, R. Wesley. A comparison of the spatiotemporal parameters, kinematics, and biomechanics between shod, unshod, and minimally supported running as compared to walking. Physical therapy in sport, v. 12, n. 4, p. 151-163, 2011.

MILNER, Clare E. e colaboradores. Biomechanical factors associated with tibial stress fracture in female runners. Medicine \& science in sports \& exercise, v. 38, n. 2, p. 323-328, 2006.

RODRIGUES, Pedro; TENBROEK, Trampas; HAMILL, Joseph. Runners with anterior knee pain use a greater percentage of their available pronation range of motion. Journal of applied biomechanics, v. 29, n. 2, p. 141-146, 2013.

SAMAAN, Cynthia D.; RAINBOW, Michael J.; DAVIS, Irene S. Reduction in ground reaction force variables with instructed barefoot running. Journal of sport and health science, v. 3, n. 2, p. 143-151, 2014.

SARAGIOTTO, Bruno Tirotti e colaboradores. What are the main risk factors for running-related injuries? Sports medicine, v. 44, n. 8, p. 1153-1163, 2014.

SUN, Xiaole e colaboradores. Systematic review of the role of footwear constructions in running biomechanics: Implications for running-related injury and performance. Journal of sports science and medicine, v. 19, p. 20-37, 2020. .

THOMPSON, Melissa A.; SEEGMILLER, Jeff G.; MCGOWAN, Craig P. Impact accelerations of barefoot and shod running. International journal of sports medicine, v. 37, n. 5, p. 364-368, 2016.

WARNE, Joe $\mathrm{P}$ e colaboradores. A 4-week instructed minimalist running transition and gaitretraining changes plantar pressure and force. Scandinavian journal of medicine and science in sports, v. 24, n. 6, p. 964-973, 2014.

WARNE, Joe P.; WARRINGTON, Giles D. Four-week habituation to simulated barefoot running improves running economy when compared with shod running. Scandinavian journal of 
medicine and science in sports, v. 24, n. 3, p. 563-568, 2014.

WILLEMS, Tine Marieke e colaboradores. A prospective study of gait related risk factors for exercise-related lower leg pain. Gait and posture, v. 23, n. 1, p. 91-98, 2006.

WILLEMS, Tine Marieke e colaboradores. Relationship between gait biomechanics and inversion sprains: A prospective study of risk factors. Gait and posture, v. 21, p. 379-387, 2005.

WILLY, Richard W; DAVIS, Irene S. Kinematic and kinetic comparison of running in standard and minimalist shoes. Medicine and science in sports and exercise, v. 46, n. 2, p. 318-323, 2014.

WIT, Brigit De; CLERCQ, Dirk De; AERTS, Peter. Biomechanical analysis of the stance phase during barefoot and shod running. Journal of biomechanics, v. 33, n. 3, 269-278, 2000.

\section{Dados da primeira autora:}

Email: anaazevedo@usp.br

Endereço: Laboratório de Biomecânica - Escola de Educação Física e Esporte, Avenida Professor Mello Moraes, 65, Vila Universitária, São Paulo, SP, CEP: 05508-030, Brasil.

Recebido em: 30/06/2021

Aprovado em: 22/07/2021

\section{Como citar este artigo:}

AZEVEDO, Ana Paula da Silva e colaboradores. Efeito agudo da corrida com os pés descalço sobre as componentes anteroposterior e mediolateral da força de reação do solo. Corpoconsciência, v. 25, n. 2, p. 175-185, mai./ ago., 2021.

\section{Agradecimentos}

Os autores agradecem ao Conselho Nacional de Desenvolvimento Científico e Tecnológico (CNPq) pelo financiamento à pesquisa e à Universidade de São Paulo pelo apoio ao estudo. Adicionalmente, os autores são gratos aos participantes da pesquisa.

\section{Indicação de apoio de órgãos de fomento}

Conselho Nacional de Desenvolvimento Científico e Tecnológico (CNPq). 